\title{
Oficina sobre orientações pedagógicas no ensino superior: ação do programa pró-saúde
}

\author{
Workshop about pedagogical orientation in higher education: pro-health program action
}

\author{
Annie Pozeczek Koltermann ${ }^{1}$, Andriele Gasparetto ${ }^{2}$, Alecsandra Pinheiro Vendrusculo ${ }^{3}$, \\ Michele Rorato Sagrillo ${ }^{4}$
}

\begin{abstract}
RESUMO
Objetivo: Analisar as potencialidades e desafios no ensino de uma instituição de ensino superior de acordo com os vetores propostos pelo Projeto Pró-Saúde do Ministério da Saúde.

Materiais e Métodos: Trata-se de uma pesquisa qualitativa, em que se utilizou análise de conteúdo em grupos focais. Realizou-se, em 2009, uma oficina organizada pela comissão interna do Pró-Saúde para discutir as potencialidades e desafios de acordo com os vetores do Projeto.

Resultados: Entre as potencialidades discutidas foi constatada a estruturação das atividades, estágios e práticas curriculares da instituição integradas à realidade e necessidades da população local. Porém, a interdisciplinaridade foi apontada como um dos grandes desafios a ser implementado na área da saúde. Nenhuma evidência atingiu o estágio III, ou seja, não foi totalmente alcançada na visão destes profissionais.

Conclusão: Fazem-se necessárias constantes discussões entre docentes para que se possam atingir resultados satisfatórios, porém, sugere-se trabalhar com um número menor de participantes.
\end{abstract}

Palavras-chave: formação de recursos humanos; educação superior; educação em saúde; pesquisa interdisciplinar.

\begin{abstract}
Objective: To analyze the strengths and challenges in teaching on a higher education institution according to the vectors proposed by the Ministry of Health Pro-Health Project.

Materials and Methods: We conducted a qualitative research, in which the analysis of content in focal groups was used. In 2009, a workshop was organized by the Pro-Health's internal commission to discuss the potentialities and challenges according to the main concerns regarding the program.

Results: Among the potentialities discussed, it was highlighted the activities structure, internships and curricular practices from the institution integrated to the reality and needs of the population. However, the interdisciplinary approach has been pointed as the biggest challenge to be implemented in the health field. None of the evidence reached the stage III, which means that none were totally achieved according to the professionals.

Conclusion: There is a need for constant discussion among teachers so that they can achieve satisfactory results, however, we suggest to work with a smaller number of participants.
\end{abstract}

Keywords: human resources formation; higher education; health education; interdisciplinary research.

\footnotetext{
${ }^{1}$ Cirurgiã-dentista. Mestre em Saúde Coletiva pela Universidade do Vale do Rio dos Sinos (UNISINOS). Docente do Curso de Odontologia do Centro Universitário Franciscano (UNIFRA) e Membro da Comissão Interna do Pró-Saúde.

${ }^{2}$ Fisioterapeuta. Mestre em Ciências da Saúde pela Universidade Federal do Rio Grande (FURG). Docente do Curso de Fisioterapia da UNIFRA e Membro da Comissão Interna do Pró-Saúde.

${ }^{3}$ Fisioterapeuta. Mestre em Ciências do Movimento Humano pela Universidade Federal do Rio Grande do Sul (UFRGS). Docente do Curso de Fisioterapia da UNIFRA.

${ }^{4}$ Bióloga. Mestre em Ciências - Genética Médica pela Escola Paulista de Medicina. Docente do Curso de Biomedicina da UNIFRA e Membro da Comissão Interna do Pró-Saúde.
} 


\section{INTRODUÇÃO}

Nas últimas décadas, muitas foram as transformações na área da Saúde e de Educação, resultando numa progressiva mobilização em torno da mudança na formação das várias profissões da saúde. A implementação das diretrizes curriculares, pelo lado da educação, e a adoção da 'integralidade' como eixo orientador dos processos de formação, pelo lado da saúde, são os nortes da política interministerial (Ministérios da Saúde e da Educação) para a mudança na graduação das profissões de saúde, tendo como ideia central das propostas de mudança a aproximação da formação com as necessidades sociais ${ }^{1}$.

Dando um breve relato deste processo de transformação no Brasil, inicia-se pela Lei Orgânica da Saúde de 1990, que, em sua definição, refere a necessidade de uma política para os trabalhadores da saúde, devendo esta ter como objetivo organizar um sistema de formação em todos os níveis de ensino, além de programas de permanente aperfeiçoamento de pessoal $^{2}$.

Ainda na década de 90, o processo de implantação do Sistema Único de Saúde (SUS) caminhou pari passu à adoção de uma série de medidas governamentais voltadas para o fortalecimento da atenção básica de saúde, entendida pelo Ministério da Saúde como "um conjunto de ações, de caráter individual ou coletivo, situadas no primeiro nível de atenção dos sistemas de saúde, voltadas para a promoção da saúde, a prevenção de agravos, o tratamento e a reabilitação"3,4. Uma das estratégias criadas nesta década para organizar a atenção básica foi o Programa de Saúde da Família (PSF), hoje reorientado em estratégia (ESF), tendo como principal desafio promover a reorientação das práticas e ações de saúde de forma integral e contínua, mais próximas da família, possibilitando a melhora na qualidade de vida dos brasileiros ${ }^{5,6}$.

Tendo em vista essa evolução e aprofundamento de questões referentes à área da saúde, deliberou-se a necessidade de mudanças na formação dos profissionais da área para acompanhar essas modificações. Surgem, então, as Novas Diretrizes Curriculares Nacionais, em 2001, dos cursos de graduação da área de saúde, deliberadas pelo
Ministério da Educação, evidenciando a preocupação com o estabelecimento de uma articulação entre a educação superior e a saúde ${ }^{7}$.

Muitas discussões vêm sendo realizadas para que o processo ensino-aprendizagem tenha a participação do aluno e as práticas de ensino ocorram parte na sala de aula e parte em ambulatórios da própria instituição de ensino ou na comunidade, tentando se aproximar da realidade do paciente/usuário. Considerando-se que do total de serviços de saúde oferecidos a maioria corresponde a serviços públicos operacionalizados nos moldes do SUS, a educação nesta área focada nos parâmetros contidos no relatório de Flexner, do ano de 1910, não condiz com os princípios e as diretrizes do sistema $^{8-10}$ o que leva a uma profunda distorção da educação, formatando profissionais alheios ao sistema de saúde vigente $e^{8,11-13}$

Visando a implementação de um programa que atendesse a dicotomia existente entre formação dos profissionais de saúde e o sistema vigente, o Ministério da Saúde, por meio da Secretaria de Gestão do Trabalho e da Educação na Saúde (SGTES) e o Ministério da Educação, por intermédio da Secretaria de Educação Superior (SESu) e do Instituto Nacional de Estudos e Pesquisas Educacionais Anísio Teixeira (INEP), lançaram, em novembro de 2005, o Programa Nacional de Reorientação da Formação Profissional em Saúde (Pró-Saúde) ${ }^{14}$.

Com a implementação do Programa Pró-Saúde, buscou-se intervir no processo formativo para que a graduação deslocasse o atual eixo da formação, centrado na assistência individual e prestada em unidades especializadas, por um processo sintonizado com as necessidades sociais, levando em conta as dimensões históricas, econômicas e culturais da população. Desta forma, pretendeu-se instrumentalizar os profissionais para a abordagem dos determinantes do processo saúde-doença na comunidade e em todos os níveis do Sistema, conforme as diretrizes de universalidade, equidade e integralidade ${ }^{14}$.

Para o desenvolvimento do Pró-Saúde foram elaborados 3 eixos (Orientação Teórica, Cenários de Prática e Orientação Pedagógica) e cada um deles contem 3 vetores. Cada um dos vetores foram 
hierarquizados em outros 3 estágios que partem de uma situação mais tradicional no estágio 1 até alcançar, no estágio 3 , a situação e o objetivo desejado ${ }^{14}$. Esse artigo relata a avaliação do Eixo C: Orientação Pedagógica.

A educação dos profissionais de saúde deve ser entendida como processo permanente na vida profissional, mediante o estabelecimento de relações de parceria entre as instituições de educação, a gestão e os serviços de saúde, a comunidade, as entidades e outros setores da sociedade civil $^{14}$. Com base nesses fatos, o presente trabalho tem como objetivo apresentar os resultados de uma oficina realizada com os professores da Área da Saúde e dos cursos Serviço Social e Psicologia, de uma Instituição de Ensino Superior (IES), considerando a implementação do Projeto Pró-Saúde com base nos desafios propostos pelo Pró-Saúde Nacional no que se refere à reorientação pedagógica.

Tendo como eixo de discussão a reorientação pedagógica, e como elementos de reflexão os Vetores 7, 8 e 9 do pró-saúde elencou-se os seguintes objetivos específicos: verificar se o processo ensinoaprendizagem toma como eixo, na etapa clínica, a análise crítica da totalidade da experiência da atenção à saúde, com ênfase no componente de Atenção Básica (Vetor 7 do pró-saúde); verificar se ocorre e como ocorre a integração do ciclo básico com o profissional ao longo do curso (um dos métodos orientador da integração é a problematização) (Vetor 8 do pró-saúde); verificar se o ensino ocorre, majoritariamente, baseado na problematização em pequenos grupos, ocorrendo em ambientes diversificados com atividades estruturadas a partir das necessidades de saúde da população. Avaliação formativa e somativa, incluindo todos os aspectos da formação do estudante (conhecimento, atitudes e habilidades) (Vetor 9 do pró-saúde) ${ }^{14}$. A discussão e análise de todos estes elementos se justificam pela necessidade de transformações do processo de formação, geração de conhecimentos e prestação de serviços à população, para uma abordagem integral do processo de saúde-doença. $O$ Pró-Saúde desenvolve-se na perspectiva de que a reorientação da formação ocorra simultaneamente em distintos eixos rumo à integração entre IES e serviço público de saúde, com reflexos na formação dos trabalhadores de saúde, na produção do conhecimento e na prestação de serviços, com vistas ao fortalecimento do SUS.

Assim, este artigo teve como objetivo analisar as potencialidades e desafios no ensino de uma instituição de ensino superior de acordo com os vetores propostos pelo Projeto Pró-Saúde do Ministério da Saúde.

\section{MATERIAIS E MÉTODOS}

Este estudo trata-se de uma pesquisa descritiva, de caráter qualitativo, em que se utilizou a análise de conteúdo em grupos focais. Participaram da oficina 40 professores dos cursos da área da saúde (Biomedicina, Enfermagem, Farmácia, Fisioterapia, Nutrição, Odontologia, Terapia Ocupacional) da instituição de ensino superior (IES), além do Serviço Social e Psicologia (nesta IES fazem parte da área de humanas). A organização foi baseada nos desafios propostos pelo Pró-Saúde Nacional e tendo como referencial teórico os desafios no que se refere à orientação pedagógica e uma auto-avaliação do grupo multidisciplinar de professores da IES em questão. A Oficina relatada nesta pesquisa ocorreu nas dependências da IES.

O pró-saúde é dividido em três grandes eixos sendo que o Eixo $C$ (norteador da atividade que será descrita neste artigo) trabalha com os Vetores 7 (Análise crítica de Atenção Básica), 8 (Integração do ciclo básico/ciclo profissional) e 9 (Mudança Metodológica).

Em um primeiro momento foi apresentado o projeto pró-saúde para os 120 professores da IES para melhor apropriação sobre o tema abordado. Em seguida estes foram subdivididos em três oficinas, cabendo a oficina do Eixo C 40 professores nos quais se propôs metodologias com o propósito de discutir e avaliar as potencialidades e desafios seguindo os objetivos específicos propostos para cada vetor do eixo C.

A divisão dos grupos foi aleatória, uma vez que cada docente recebia uma cor na entrada ao evento que posteriormente serviria para a divisão dos três grupos nas três oficinas. Este estudo abordará uma destas oficinas que contou com a participação de 40 professores. Após essa divisão foi apresentado ao 
TABELA 1 - Relatório da oficina do Eixo C - Vetor 7.

\begin{tabular}{|c|c|c|c|}
\hline Evidência - Vetor 7 & Estágio I, II ou III & Potencialidades & Desafios \\
\hline $\begin{array}{l}\text { A escola enfatiza o aprendizado } \\
\text { prático, no qual o estudante, } \\
\text { com participação dos } \\
\text { profissionais dos serviços e } \\
\text { professores, adquire } \\
\text { responsabilidade progressiva e } \\
\text { crescente, tendo em vista a } \\
\text { permanente melhoria dos } \\
\text { serviços à população? }\end{array}$ & II & $\begin{array}{l}\text {-incentivo da instituição no } \\
\text { processo } \\
\text {-integração entre os cursos } \\
\text { (docentes e discentes) } \\
\text {-infra-estrutura } \\
\text {-estágios, atividades e ou práticas } \\
\text { integradas. }\end{array}$ & $\begin{array}{l}\text {-efetivar a interdisciplinaridade } \\
\text {-"momentos" para integração dos } \\
\text { professores } \\
\text {-operacionalização do processo de } \\
\text { integração de algum serviço de } \\
\text { saúde, tendo o profissional do serviço } \\
\text { um facilitador. } \\
\text {-conhecimento e comprometimento } \\
\text { dos docentes e profissionais de } \\
\text { saúde com o sistema }\end{array}$ \\
\hline $\begin{array}{l}\text { Os estudantes, na prática da } \\
\text { atenção à saúde, procedem à } \\
\text { análise integral e sistemática do } \\
\text { processo saúde-doença nas } \\
\text { suas várias dimensões } \\
\text { (individual, familiar e coletiva) } \\
\text { por meio da problematização de } \\
\text { situações vivenciadas? }\end{array}$ & II & $\begin{array}{l}\text {-espaços para a prática: estágios, } \\
\text { serviço integrado e núcleos de } \\
\text { prática. } \\
\text {-contato direto e precoce do } \\
\text { acadêmico com o usuário } \\
\text {-integração das disciplinas do } \\
\text { semestre em torno de um tema } \\
\text { social e de saúde } \\
\text {-estímulo para reflexão do } \\
\text { processo ensino-aprendizagem } \\
\text { em saúde }\end{array}$ & $\begin{array}{l}\text {-adaptação dos professores ao novo } \\
\text { sistema de interdisciplinaridade } \\
\text {-mudança da visão do aluno quanto à } \\
\text { prática de saúde coletiva bio-psico- } \\
\text { social }\end{array}$ \\
\hline
\end{tabular}

Estágio II: Algumas disciplinas de aplicação clínica do curso proporcionam oportunidade para análise crítica da organização do serviço.

grupo todos os vetores que contemplam esse eixo e suas evidências. Foi entregue para cada grupo perguntas pertinentes de cada vetor nos quais os professores precisariam respondê-las para posteriormente discuti-las com o grupo maior.

Para cada um dos vetores utilizaram-se evidências elaboradas pelo Plano Nacional e contidos no Projeto Pró-Saúde para serem debatidas e consequentemente gerando potencialidades e desafios para cada uma delas, bem como situando-se o estágio em que a Instituição estaria inserida, conforme o Programa Nacional $^{14}$.

A atividade proposta aos três grupos de docentes teve duração de cinco horas e para cada um destes foi solicitado o estudo de cada vetor.

O Vetor 7 era constituído de 2 evidências:

- A escola enfatiza o aprendizado prático, no qual o estudante, com participação dos profissionais dos serviços e professores, adquire responsabilidade progressiva e crescente, tendo em vista a permanente melhoria dos serviços à população?

- Os estudantes, na prática da atenção à saúde, procedem à análise integral e sistemática do processo saúde-doença nas suas várias dimensões (individual, familiar e coletiva) por meio da problematização de situações vivenciadas?

O Vetor 8 apresentava duas evidências para serem debatidas:

- A programação do ciclo básico, além da essência conceitual de cada disciplina biomédica, leva em conta a realidade da prática clínica, incorporando o pessoal dos serviços e os professores do ciclo profissional no processo de planificação curricular?

- A escola adota algum esquema modularizado de organização dos conteúdos que facilite os processos de interdisciplinaridade e integração de conhecimentos?

O último Vetor a ser discutido nesse espaço de avaliação foi o 9, onde discutiram-se 3 evidências:

- O ensino-aprendizagem se desenvolve em pequenos grupos? Estimula-se a busca de informações e o processo de auto-aprendizagem, envolvendo situações diversificadas (resolução de problemas, análises de casos, simulações, dentre outras), e em distintos cenários (Escola, SUS, comunidade etc.), estimulando capacidades cognitivas, psicomotoras e afetivas?

- Estímulo à discussão crítica e reflexiva sobre as práticas, por todos os participantes no processo (professores,profissionais dos serviços, estudantes, da gestão das organizações envolvidas? 
TABELA 2 - Relatório da oficina do Eixo C - Vetor 8.

\begin{tabular}{|c|c|c|c|}
\hline Evidência - Vetor 8 & Estágio I, II ou III & Potencialidades & Desafios \\
\hline $\begin{array}{l}\text { A programação do ciclo básico, além } \\
\text { da essência conceitual de cada } \\
\text { disciplina biomédica, leva em conta a } \\
\text { realidade da prática clínica, } \\
\text { incorporando o pessoal dos serviços e } \\
\text { os professores do ciclo profissional no } \\
\text { processo de planificação curricular? }\end{array}$ & II & $\begin{array}{l}\text {-presença dos profissionais e } \\
\text { alunos da UNIFRA nos serviços e } \\
\text { estágios supervisionados } \\
\text {-bons campos de prática } \\
\text {-experiência profissional dos } \\
\text { professores aliada à experiência } \\
\text { acadêmica } \\
\text {-integração das disciplinas do } \\
\text { semestre em torno de um tema } \\
\text { social e de saúde }\end{array}$ & $\begin{array}{l}\text { - incorporar os profissionais } \\
\text { do serviço das unidades } \\
\text { básicas de saúde } \\
\text { - modificar a visão curativa }\end{array}$ \\
\hline $\begin{array}{l}\text { A escola adota algum esquema } \\
\text { modularizado de organização dos } \\
\text { conteúdos que facilite os processos } \\
\text { de interdisciplinaridade } \\
\text { integralização de conhecimentos? }\end{array}$ & II & $\begin{array}{l}\text {-ausência de departamentalização } \\
\text {-existência do serviço integrado } \\
\text { em saúde } \\
\text {-grade curricular construída } \\
\text { direcionada à proposta } \\
\text {-utilização de outros sistemas de } \\
\text { integração dos conteúdos que não } \\
\text { os sistemas de módulos }\end{array}$ & $\begin{array}{l}\text {-criar espaço para integrar os } \\
\text { professores } \\
\text {-implementação de } \\
\text { disciplinas, atividades, TFGs } \\
\text { e projetos que integrem corpo } \\
\text { docente e discente } \\
\text {-avaliação dos diferentes } \\
\text { processos }\end{array}$ \\
\hline
\end{tabular}

Estágio II: Disciplinas/atividades integradoras, ao longo dos primeiros anos, em algumas áreas.

- Avaliação participativa do aluno com metodologias quali-quantitativas e multirreferências, incluindo a do currículo desenvolvido?

Após a realização destas discussões, fez-se a exploração do material e posterior análise de conteúdos através da categorização dos resultados. A partir disso, categorizou-se os resultados partindo de seus próprios vetores, sendo aqui chamados de vetor 7 , vetor 8 e vetor 9. Os resultados são aqui apresentados de forma descritiva com a utilização de tabelas para facilitar a visualização e discutidos com literatura atual e clássica. Ao final da atividade, os resultados de todas as oficinas foram apresentados aos docentes da instituição.

\section{RESULTADOS E DISCUSSÃO}

Com base nas discussões ocorridas na Oficina foram elaboradas tabelas que contém os resultados de cada evidência analisada. Quando analisadas as potencialidades da IES frente às questões abordadas no Vetor 7 (tabela 1), foram relatadas a estruturação das atividades, estágios e práticas curriculares da instituição integradas à realidade da população, visando a permanente melhoria dos serviços, com total incentivo da instituição neste processo.

Esta estrutura curricular permite que o acadêmico tenha contato direto e precoce com o usuário, o que possibilita estímulo para reflexão do processo ensinoaprendizagem em saúde, de acordo com o modelo pedagógico que orienta o aprendizado baseado na prática, que deve ocorrer de forma dinâmica através da ação-reflexão-ação ${ }^{14}$.

Porém, percebe-se como principal desafio no vetor 7 a interdisciplinaridade, sendo este o grande desafio do ensino de forma geral, composto historicamente pela exagerada especialização do saber, trazendo para o debate coletivo questionamentos sobre o sentido e a pertinência das colaborações entre as disciplinas, visando assim, um conhecimento que enfatiza o saber sobre o homem e sobre a sociedade ${ }^{15}$. A interdisciplinaridade é considerada uma inter-relação e interação das disciplinas a fim de atingir um objetivo comum $^{15}$. Baseado neste contexto percebe-se a necessidade de espaços para integração docente, iniciando-se desta forma o processo de conjugação de saberes, que posteriormente poderá ser levado à prática através das próprias atividades curriculares já previstas pela instituição, ou, partindo a integrar vários conhecimentos em prol de um objetivo em comum: o desenvolvimento social.

Dentre as evidências debatidas no vetor 8 (tabela 2) está a integração do ensino, prática clínica, profissionais dos serviços e docentes, sendo esta integração indispensável para a formação de recursos 
TABELA 3 - Relatório da oficina do Eixo C - Vetor 9.

\begin{tabular}{|c|c|c|c|}
\hline Evidência - Vetor 9 & Estágio I, II ou III & Potencialidades & Desafios \\
\hline $\begin{array}{l}\text { O ensino-aprendizagem se desenvolve } \\
\text { em pequenos grupos? Estimula-se a } \\
\text { busca de informações e o processo de } \\
\text { auto-aprendizagem, envolvendo situações } \\
\text { diversificadas (resolução de problemas, } \\
\text { análises de casos, simulações, dentre } \\
\text { outras), e em distintos cenários (Escola, } \\
\text { SUS, comunidade etc.), estimulando } \\
\text { capacidades cognitivas, psicomotoras e } \\
\text { afetivas? }\end{array}$ & II & $\begin{array}{l}\text {-aprendizagem diversificada } \\
\text {-está em desenvolvimento } \\
\text { em algumas áreas "estágio" } \\
\text {-CORES }\end{array}$ & $\begin{array}{l}\text {-ter pequenos grupos para } \\
\text { trabalho } \\
\text {-adequar a avaliação } \\
\text { considerando aspectos } \\
\text { cognitivos, crítico-reflexivos e } \\
\text { éticos } \\
\text {-despertar o interesse do } \\
\text { aluno pela sistemática de } \\
\text { aprendizagem }\end{array}$ \\
\hline $\begin{array}{l}\text { Estímulo à discussão crítica e reflexiva } \\
\text { sobre as práticas, por todos os } \\
\text { participantes no processo (professores, } \\
\text { profissionais dos serviços, estudantes, } \\
\text { usuários) visando à transformação da } \\
\text { própria prática e da gestão das } \\
\text { organizações envolvidas? }\end{array}$ & II & $\begin{array}{l}\text {-faz parte da visão } \\
\text { institucional } \\
\text {-acontece entre os } \\
\text { professores } \\
\text {-realização de eventos que } \\
\text { propiciem a participação de } \\
\text { todos }\end{array}$ & $\begin{array}{l}\text {-incluir o serviço e o usuário } \\
\text { no processo } \\
\text {-otimizar espaço e tempo para } \\
\text { estimular a discussão crítica e } \\
\text { reflexiva } \\
\text {-carência de um "momento de } \\
\text { integração" entre professores, } \\
\text { alunos e servidores }\end{array}$ \\
\hline $\begin{array}{l}\text { Avaliação participativa do aluno com } \\
\text { metodologias quali-quantitativas e } \\
\text { multireferências, incluindo a avaliação do } \\
\text { currículo desenvolvido? }\end{array}$ & 1 & $\begin{array}{l}\text { - respaldo institucional para } \\
\text { este tipo de avaliação CPA }\end{array}$ & $\begin{array}{l}\text {-incluir o aluno neste tipo de } \\
\text { avaliação } \\
\text {-estimular a crítica construtiva } \\
\text {-número de alunos para este } \\
\text { tipo de avaliação }\end{array}$ \\
\hline
\end{tabular}

Estágio I. Ensino centrado no professor, realizado fundamentalmente por meio de aulas expositivas para um grande número de estudantes.

Estágio II. Ensino que pode incluir inovações pedagógicas em caráter experimental, mas restritas a certas disciplinas. Ensino realizado, em geral, com pequenos grupos de estudantes.

humanos na saúde, tendo como pano de fundo o processo de implantação e consolidação do SUS. Tendo em vista a mudança na base conceitual, política e operacional da educação em saúde, verifica-se a necessidade de reflexão sobre as transformações que se operam no nível da formação profissional, no que diz respeito à reorganização das instituições de ensino superior, às oportunidades de reorientação de novos modelos pedagógicos que contemplem a excelência técnica e a relevância social e à identificação das competências do setor da saúde e da educação no ordenamento dos profissionais na direção da consolidação do SUS ${ }^{16}$.

Nesta perspectiva, a IES traz como potencialidades os campos de Estágio, que integram docentes, discentes e profissionais da atenção básica, atuando na integração das disciplinas com questões de relevância social, aliando a experiência profissional dos professores à experiência acadêmica. Porém, como desafios predominam a dificuldade de incorporar os profissionais do serviço das unidades básicas de saúde a este processo de integração, sendo que a visão curativa dentro da saúde ainda se constitui em um forte paradigma a ser vencido.

A interdisciplinaridade e integralização de conhecimentos também constituiu evidência em debate no Vetor 8 (tabela 2), sendo potencialidades neste sentido a ausência de departamentalização na instituição, a existência de serviço integrado em saúde, grade curricular construída direcionada à proposta e utilização de outros sistemas de integração dos conteúdos que não os sistemas de módulos.

Considerando que a interdisciplinaridade é fundamentalmente um processo e uma filosofia de trabalho que entra em ação na hora de enfrentar os problemas e questões que preocupam cada sociedade $^{17}$, mais uma vez destaca-se a necessidade de um campo de debate, onde as questões 
interdisciplinares da saúde devem ser consideradas, já que a complexidade se acentua quando procuramos entender saúde no âmbito coletivo, cujo objeto envolve o biológico e o social, o indivíduo e a comunidade e ainda, a política social e econômica ${ }^{15}$.

Quando discutidas as três evidência do vetor 9 (tabela 3) entre os docentes, nota-se que muitas foram as potencialidades da Instituição e muitos também foram os desafios a enfrentar.

Ao analisar a primeira evidência que traz alguns questionamentos como estímulo de busca de informações e autoaprendizagem envolvendo situações diversificadas e distintos cenários, além de estímulo a capacidade cognitiva, psicomotora e afetiva, os docentes apontaram as potencialidades institucionais como uma aprendizagem diversificada e a criação da Coordenadoria de Atenção ao Estudante (CORES), que tem o objetivo de prestar assistência aos estudantes com vistas a sua integração acadêmica, científica e social. Isso se efetivará por meio de ações de acolhimento, apoio psicopedagógico na organização, gestão das aprendizagens e métodos de estudo e promoção da adaptação e do sucesso acadêmico. Os desafios dessa evidência permearam na necessidade de existirem grupos menores de trabalho, avaliações adequadas aos aspectos docentes e a necessidade de despertar no aluno o interesse pela aprendizagem. Para Falcón et al. ${ }^{18}$ é missão da Universidade cultivar o prazer pelo saber, transformar a curiosidade em investigação científica, entre outros, para que assim consiga-se aprender e educar para a complexidade do mundo. Para isso, faz-se necessária a busca por diferentes estratégias metodológicas e diferentes campos de práticas, como discutido entre os docentes.

Sobre o estímulo à discussão crítica e reflexiva sobre as práticas, os docentes afirmaram ser esta uma visão da própria instituição, porém, afirmaram existir ainda uma carência em momentos de integração entre docentes, alunos e servidores e ainda incluir o serviço e o usuário no processo educacional. Ceccim \& Feuerwerker ${ }^{8}$ discutem a necessidade de aproximação entre ensino - serviço - gestão - controle social para assim qualificar a formação dos trabalhadores para as ser desenvolvida e aprimorada, pois irá possibilitar a reais necessidades de saúde, já que Falcón et al. ${ }^{18}$ afirma que existe, ainda, inadequação entre a formação dos profissionais de saúde e a realidade. Aproximando os atores da educação, a formação se relacionará mais facilmente com o movimento de transformação que a sociedade vive. Ainda, discute-se que "o processo educativo é, sobretudo, uma relação entre seres humanos, relação que está permeada pelo conjunto de valores, práticas sociais, costumes e tradições que fazem parte da formação cultural” ${ }^{19}$

A última evidência do vetor 9 discute se existe avaliação participativa do aluno com metodologias qualiquantitativas e multireferências, incluindo a avaliação do currículo desenvolvido. Os docentes consideraram essa evidência a que apresenta mais necessidade de desenvolvimento e evolução. Foram apontadas como potencialidades novamente 0 respaldo da IES em questão e como desafios a necessidade de inserção do aluno nesse processo, além de torná-lo um sujeito crítico. Salienta-se a necessidade de estimulo à formação de caráter reflexivo e crítico dos educandos, preparando-os, assim para as diferentes formas de enfrentamento dos problemas junto ao seu educador, tanto durante as aulas quanto na vida profissional futura $^{20}$.

\section{CONSIDERAÇÕES FINAIS}

Com base nesses dados percebe-se o expressivo resultado obtido após esta discussão entre docentes de nove cursos de graduação da IES, contemplando trocas importantes entre diferentes profissionais, de diferentes cursos e com diferentes vivências. Através desses resultados será possível pensar estratégias que superem os desafios apontados como fragilidades na prática educacional. Percebe-se que das 7 evidências lançadas entre os 3 vetores, nenhuma atingiu o estágio III, ou seja, nenhuma alcançou o objetivo desejado pelos docentes. Seis delas foram classificadas como pertencentes ao Estágio II e uma no Estágio I. Isso significa que em todas as evidências avanços ainda devem ser alcançados para a melhoria da orientação pedagógica. Seguindo esse critério a avaliação participativa do aluno é a evidência que mais necessita ação-reflexão-ação, proporcionando o diálogo como 
uma prática essencial no processo, levando os docentes e discentes a discutir a realidade, buscando assim formar um profissional com qualidade técnicohumanística, ética e também política. Desta forma deverá favorecer a realização de diferentes técnicas e procedimentos como a observação sistemática, análise da realidade, o exercício da solução de problemas, além de introduzir precocemente os alunos à realidade de saúde local e regional considerando as atividades práticas, diversificar os cenários de aprendizagem buscando propiciar aos acadêmicos o conhecimento do sistema e das políticas de saúde, primar pela interdisciplinaridade na abordagem e na construção dos conteúdos, possibilitar a avaliação participativa, com troca de experiências entre alunos, professores e profissionais dos serviços.

Sendo assim, entende-se que embora identificadas várias potencialidades, desafios ainda permeiam a prática educacional dos discentes e docentes no ensino superior. Fazem-se necessárias discussões periódicas entre os docentes para que se consiga a busca por melhores resultados na educação superior, porém, sugere-se trabalhar com um número menor de participantes em cada grupo, como preconizado neste tipo de metodologia aplicada.

\section{REFERÊNCIAS}

1. Albuquerque VS, Giffin KM. Globalização capitalista e formação profissional em saúde: Uma agenda necessária ao ensino superior. Trab Educ Saúde. nov. 2008/ fev. 2009; 6(3):519-37.

2. Brasil. Lei Orgânica de Saúde no. 8.080 , de 19 de setembro de 1990. Dispõe sobre as condições para a promoção, proteção e recuperação de saúde, a organização e o funcionamento dos serviços correspondentes e da outras providencias. 1990; Diário Oficial da União.

3. Brasil. Constituição de 1988. Constituição da República Federativa do Brasil. Brasília: Senado Federal; 1988.

4. Escorel S, Giovanella L, Mendonça MHM, Senna MCM. O Programa de Saúde da Família e a construção de um novo modelo para a atenção básica no Brasil. Rev Panam Salud Publica/Pan Am J Public Health. 2007; 21(2/3):164 76.

5. Brasil. Ministério da Saúde. Departamento de Atenção Básica. Guia prático do Programa de Saúde da Família. Brasília: Ministério da Saúde; 2001.
6. Brasil. Ministério da Saúde. Programa Saúde da Família. Equipes de Saúde Bucal: Projetos, programas e relatórios. Brasília: Ministério da Saúde; 2002.

7. Câmara de Educação Superior. Conselho Nacional de Educação. Resolução CNE/CES 3, 2002; 19 de fev.[capturado 2008 jun 2]; Disponível em: http://portal.mec.gov.br/cne/arquivos/pdf/CES032002.pdf.

8. Ceccim RB, Feuerwerker LCM. O quadrilátero da formação para a área da saúde: ensino, gestão, atenção e controle social. Physis. 2004; (1):41-65

9. Mendes EV. Uma agenda para a saúde. São Paulo: Hucitec; 1998.

10. Feuerwerker LCM. Estratégias para a mudança da formação dos Profissionais de saúde. Caderno CE. 2001;(2): 11-23.

11. Brasil. Ministério da Saúde. Secretaria de Gestão do Trabalho e da Educação na Saúde. Departamento de Gestão da Educação na Saúde. Rev-SUS Brasil: cadernos de textos. Brasília: Ministério da Saúde; 2004.

12. Schaedler LI. Sistema Único de Saúde como Rede em Prática Pedagógica. In: Brasil, Ministério da Saúde. VERSUS Brasil: Caderno de Textos. Brasília: Ministério da Saúde; 2004.

13. Cotta RMM, Gomes AP, Maia TM, Magalhães KA, Marques ES, Siqueira-Batista R. Pobreza, Injustiça, e Desigualdade Social: repensando a formação de Profissionais de Saúde. Rev Brasileira de Educação Médica. 2007, (3):278-86.

14. Brasil. Ministério da Saúde. Ministério da Educação. Programa Nacional de Reorientação da Formação Profissional em Saúde - Pró-Saúde: objetivos, implementação e desenvolvimento potencial. Brasília: Ministério da Saúde; 2007.

15. Vilela EM, Mendes IJM. Interdisciplinaridade e Saúde: estudo bibliográfico. Rev Latino-am Enfermagem. 2003 jul/ago; 11 (4):525-31.

16. Costa RKS, Miranda FAN. Formação profissional no SUS: oportunidades de mudanças na perspectiva da Estratégia de Saúde da Família. Trab Educ Saúde. nov. 2008/ fev. 2009; 6(3):503-17.

17. Torres JS. Globalização e interdisciplinaridade: o currículo integrado. Porto Alegre: Artmed; 1998.

18. Falcón GS, Erdmann AL, Meirelles BHS. A Complexidade na educação dos profissionais para o cuidado em saúde. Texto \& Contexto Enferm. 2006; 15(2):343-51.

19. Pereira SE. Contribuições para um planejamento educacional em ciências da saúde com estratégias inovadoras de ensino-aprendizagem. Com Ciências Saúde. 2007, 18(1):33-44.

20. Freire $P$. Pedagogia do oprimido. $28^{a}$ ed. Rio de Janeiro: Paz e Terra; 2000.

\section{Endereço para correspondência}

Annie Pozeczek Koltermann

Av. Medianeira 1899, apto 301

Santa Maria/RS - CEP 97060-003

Telefone: +55 5533177505 e +55 5599744002

E-mail:anniepk@gmail.com 